\title{
Perceptions of Psychological Momentum of Elite Soccer Players
}

Athalie J. Redwood-Brown and Caroline D. Sunderland,

Nottingham Trent University

United Kingdom

\author{
Antoinette M. Minniti \\ American Psychological Association \\ United States of America \\ Peter G. O’Donoghue \\ Cardiff Metropolitan University \\ United Kingdom
}

Correspondence concerning this article should be addressed to Athalie.Redwoodbrown@ntu.ac.uk 


\begin{abstract}
The purpose of this research was to investigate elite soccer players' perceptions and experiences of psychological momentum (PM) using a mixed methodological approach. Specifically, by comparing responses, the study aimed to provide coaches with a more appropriate method for collecting PM data. Interviews and focus groups were conducted with 10 English Academy, elite male soccer players. In addition, 75 professional male soccer players completed a 49-item measure about their experiences of PM. Interviews and focus groups were manually analysed using a combination of inductive and deductive approaches with Chi-square tests used to assess differences between responses to the PM measure. The majority of questionnaire responses were supported by themes highlighted by players interviewed. Scoring and conceding goals were the most frequently reported match variables associated with positive and negative PM, respectively. In addition, 'feeling confident', 'having a positive attitude' and 'being cohesive as a team' were important aspects of positive PM. A 'perceived lack of ability' and 'feeling anxious' were the most frequently reported experiences of negative PM. The similarity of results from both methods support the measure as a useful tool for coaches to collect data pertaining to players' experiences and perceptions of PM. Overall, findings suggest that PM is a complex (dynamic) process whereby triggers, characteristics, and consequences can hardly be separated. By understanding players' experiences and perceptions of PM, coaches may incorporate specific training methods to help players maximise positive PM, reduce negative PM as well as develop strategies to optimise PM.
\end{abstract}




\section{Introduction}

In sport, one of the most common psychological concepts discussed yet poorly understood is the concept of momentum (Taylor \& Demick, 1994). Coaches commonly admit to adjusting their line-ups, game strategies, and tactics to take advantage of athletes with apparent momentum (Perreault, Vallerand, Montgomery, \& Provencher, 1998). This may be especially pertinent in soccer where the outcome of the match has large financial implications (Hoffmann, Ging, \& Ramasamy, 2002). The term "momentum" is frequently used to describe where the sequence of scoring or winning (success) has an influence on future performance success (Briki, Den Hartigh, Markman, Micallef, \& Gernigon, 2013; Gernigon, Briki, \& Eykens, 2010; Iso-Ahola \& Dotson, 2014; Taylor \& Demick, 1994; Vallerand, Colavecchio, \& Pelletier, 1988). In contrast, "psychological momentum” (PM) is a term used in sport psychology that captures not only the success - future performance relationship, but also shifts in affect, cognition and behaviour in either a positive or negative direction in athletes, coaches and fans (Jones \& Harwood, 2008; Markman \& Guenther, 2007; Perreault et al., 1998; Taylor \& Demick, 1994; Vallerand et al., 1988).

There seems to be a strong belief that PM is an important determinant of success in sporting contests, even though there is both evidence for (Cornelius, Silva, Conroy, \& Petersen, 1997; Jones \& Harwood, 2008) and against (Koehler \& Conley, 2003) a positive relationship between PM and sport performance. Research suggests that perceptions of PM exist and alter in response to gaining or losing ground in a competition (i.e., scoring or conceding) (Briki, Den Hartigh, Hauw, \& Gernigon, 2012; Eisler \& Spink, 1998; Iso-Ahola \& Dotson, 2014; Moesch \& Apitzsch, 2012; Perreault et al., 1998; Stanimirovic \& Hanrahan, 2004) and therefore might be a possible explanation why a player's performance changes in relation to the score line. Therefore, in this paper the definition of PM as a "perception that the actor is progressing towards his/her goal" (Vallerand et al., 1988, p. 94) will be used, as it 
considers the perception of the performer as well as the act of scoring which has been shown to be a key determinant of PM in soccer (Higham, Harwood, \& Cale, 2005; Jones \& Harwood, 2008).

Several conceptual models have been proposed to explain PM. The earliest was the antecedents-consequences model (AC) proposed by Vallerand et al. (1988), which was followed by the multi-dimensional model (MD) (Taylor \& Demick, 1994), and the projected performance model (PP) (Cornelius et al., 1997). More recently, Gernigon et al. (2010) proposed that PM could be described as a dynamical system, where the system or team, in the case of most sporting examples does not vary as a function of one or two independent variables (e.g., triggers) but as a function of its preceding state (e.g., whether the team is in a stable or unstable state). The view is supported by qualitative research (Briki, Den Hartigh, Hauw, \& Gernigon, 2012; Jones \& Harwood, 2008) which reported a number of PM variables as both determinants and consequences of PM states. Such variables pertaining to PM have generally been classed as internal (complacency, psychological states, fatigue), environmental (scoring, dramatic actions, opponents) or social (team cohesion, staff influences, crowd influences) (Taylor \& Demick, 1994) which influence performance either independently or as a complex interaction depending on the stability of the system.

Employing the dynamical systems theory, Gernigon et al. (2010) examined the influence of increasing (i.e., positive momentum) versus decreasing (i.e., negative momentum) scoring sequences on several psychological components (e.g., self-confidence, competitive anxiety, achievement goals) during observed tennis matches. Negative events were found to have a more powerful psychological impact than positive events of the same value. Both somatic and cognitive anxiety were lower in the increasing scenario than the decreasing, whereas the reverse was found for self-confidence. Previous studies have shown that moving towards (or away from) a goal has positive (or negative) consequences on 
emotions such as competitive anxiety and self-confidence (as well as collective efficacy) (Briki, Den Hartigh, Bakker, \& Gernigon, 2012; Gernigon et al., 2010; Den Hartigh, Gernigon, Van Yperen, Marin, \& Van Geert, 2014; Stanimirovic \& Hanrahan, 2004).

To test the dynamical systems theory in practice, Briki et al. (2013) extended Gernigon et al.’s (2010) findings using actual cyclists (as opposed to observers) competing against each other on home trainers. Bogus feedback was given showing either an ascending performance scenario (i.e., positive momentum) or a descending performance scenario (i.e., negative momentum). Supporting the findings of Gernigon et al. (2010), the negative momentum scenario elicited an abrupt decrease in PM perceptions, whereas in the positive scenario PM perceptions increased gradually. This finding indicates that negative momentum was entered relatively rapidly compared to positive momentum and, thus, from an applied perspective it would be essential to develop strategies to overcome negative PM or create positive PM.

The most recent study to attempt to support the dynamical system theory was conducted by Den Hartigh et al. (2014) and was the first study to consider the dynamics of team momentum. Their results revealed that exerted effort, collective efficacy and task cohesion decreased dramatically in the negative momentum scenario compared to the positive scenario. Interestingly, lagging behind at the start of the race (positive momentum scenario) did not have as much of a negative impact on task cohesion and collective efficacy as losing the lead did when being close to achieving victory (negative momentum scenario) (Den Hartigh et al., 2014). This may explain why soccer teams suffer dramatic performance decreases after a score against the run of play (Higham et al., 2005).

In summary, these studies (Briki et al., 2013; Gernigon et al., 2010; Den Hartigh et al., 2014) have shown that progressing toward or away from victory causes changes in various psychological and behavioural states. However, with relatively few variables 
investigated and a focus on the characteristics of the PM state, this previous work provides only a brief overview of the experiences and perceptions of PM.

Crust and Nesti (2006) suggested that qualitative methods should be employed to examine individual perceptions and experiences of PM to better understand the psychological momentum phenomenonology. To address this, Jones and Harwood (2008) interviewed university soccer players to investigate triggers, experiences, and potential consequences (e.g., behavioural affect) related to PM, and strategies used to control perceptions of PM within competitive soccer matches. A high level of confidence, scoring a goal, and seeing negative body language in opponents were all factors that were perceived as key triggers of positive PM, whilst negative momentum was triggered by incidents where opponents played to their strengths or maintained possession (Jones \& Harwood, 2008). More recently, Briki, Den Hartigh, Hauw, and Gernigon (2012) interviewed senior table tennis players and swimmers regarding their experiences of both positive and negative momentum. Although similarities in player responses between Jones and Harwood (2008) and Briki, Den Hartigh, Hauw, and Gernigon (2012) were found, it was highlighted that PM experiences could have been dependent on the context of the situation which can differ depending on the sport in question (Higham et al., 2005). For example, Briki, Den Hartigh, Hauw, and Gernigon (2012) found that reducing effort (i.e., coasting) when winning had more of a physiological impact on swimmers than the table tennis players who reported a greater motivational impact than swimmers. It would appear that some sports may have greater demands in some areas than others (e.g., physical versus psychological), and although some PM experiences are standard (e.g., self-confidence) others may be reflective of the sport and age group being analysed.

Qualitative methods were also used by Moesch and Apitzsch (2012) who interviewed coaches about their perceptions of PM. Coaches reported that they perceived positive PM to be associated with joy, happiness, and pride in their athletes and negative PM with anxiety 
and stress, which is similar to players' perceptions in the previous studies. Confidence (or lack of) was also a major factor perceived to lead to continued positive performance (or negative performance) and increased (or decreased) effort in their players. Unlike the findings of both Jones and Harwood (2008) and Briki, Den Hartigh, Hauw, and Gernigon (2012) coaches in Moesch and Apitzsch's (2012) study perceived that players' opponents had a large impact on their players, influencing both positive and negative PM. It is perhaps not only the sport but also the sex of the individuals that may affect perceptions of PM as the coaches interviewed by Moesch and Apitzsch (2012) were all coaches of female athletes.

Although there is no single best method for investigating PM, manipulating perceived progress toward or away from a goal to simulate positive or negative PM may lack ecological validity, as progress is not based on actual performance. Lab-based studies are also limited with regards to the depth of information reported about positive and negative PM scenarios. As an alternative, qualitative methods are useful for exploring experiences of negative and positive PM in more depth; however, such methods have generally relied on small sample sizes. The purpose of the current study was to investigate players' experiences of psychological momentum using a mixed methodological approach. Due to the depth of information available from qualitative methods, interviews focused on both the triggers and characteristics of PM as well as strategies used to gain positive PM and overcome negative PM. It was proposed that if both methods produced similar results, the quantitative methods may provide coaches with a more proximal (e.g., closer to the game) way of collecting PM data.

\section{Method}

\section{Participants}

Elite male soccer players, with at least two years of experience in a professional academy, were recruited to participate in interviews and focus groups and/or subsequent 
completion of a questionnaire to assess perceptions of PM. Athletes were considered elite if they held a professional contract with their club (Ford \& Williams, 2011). Ten English U18 League One academy players (six defenders, three midfield players, and one striker) were recruited for interviews, focus groups, and completion of the questionnaire $\left(M_{\mathrm{age}}=17.10\right.$ years, $S D=0.56$ years). Four additional teams from professional English Soccer Leagues were recruited to complete the questionnaire only $(N=65)$, resulting in a total of 75 respondents (across all positions) for the questionnaire data $\left(M_{\mathrm{age}}=22.8\right.$ years, $S D=3.12$ years).

Adult players gave informed consent, and for U18 athletes this was obtained both through their club contracts and via opt-out consent from each player's respective parent or guardian. Prior to data collection, participant information sheets were distributed and individuals were briefed about the research purpose, data confidentiality, and their right to withdraw. Ethical approval was granted by the University's Ethics Committee.

\section{Procedure}

The data collection design was developed to minimise response bias (i.e., either group of participants conferring with each other and/or in between matches). For the players completing the interview, focus groups, and questionnaire $(N=10)$, data was collected over six weeks to allow a two-week gap between each data collection point. Individual semistructured interviews, with an identical set of questions asked in a similar manner (Hanton, Fletcher, \& Coughlan, 2005) were conducted first to investigate players' perceptions and experiences of PM in soccer.

Interviews included main questions to initiate conversation, and probe questions to encourage elaboration (e.g., asking for specific examples where these situations had occurred in matches). Focus groups were conducted two weeks after completion of initial interviews, with a convenience sub-sample of players in groups of two to four, follow-up questions were 
used as a means to further explore emergent themes and to clarify, summarise, and ensure the interviewer accurately interpreted the responses. The process facilitated exploration of identified themes and relevant issues and ensured fluent discussion and richness of the data (Patton, 2002). A full copy of the interview guide can be requested from the first author.

Questionnaires $(N=75)$, were administered two weeks after the completion of focus groups during the respective clubs' feedback sessions. The quantitative measure allowed data to be collected within 48 hours of the match being played, with the potential to reduce memory decay that could occur if data collection were delayed. The PM measure (further discussed below) was administered by either the Head of Performance Analysis from each respective club, or one of the key coaching team who had been involved in the development of the measure. Administration guidelines were provided to ensure consistency across teams and to ensure players completed the questionnaire without conferring with teammates. Due to club confidentiality issues, responses were recorded only according to position type and club.

\section{Instrumentation: Interviews and focus groups.}

The individual interview questions were adapted from Jones and Harwood's (2008) interview guide which, after clarifying PM, asked players about their general experiences and perceptions of PM (e.g., triggers and characteristics of PM as well as strategies used to gain or overcome PM). Using Patton's (2002) recommendations for conducting interviews, the use of clarification, elaboration, and general probes was essential for expanding on issues raised by participants. Interviews included 39 questions and lasted on average 26 minutes $(S D=3$ min) and ranged from $20-35$ minutes.

Focus groups were used to encourage further discussion amongst peers and to clarify themes that had previously emerged from individual interviews. They lasted on average 18 minutes $(S D=2 \mathrm{~min})$ and ranged from 14 to 23 minutes. No formal guide was used as participants were prompted to expand on themes emerging from the initial interviews. For 
example, players were asked to highlight specific situations that had been recalled in the first interviews and asked for elaboration and/or clarification on these themes. Interviews and focus groups, respectively, were concluded once data saturation was reached (i.e., no new information was being provided by interviewees in relation to the focus of the study) in line with recommendations of previous researchers (e.g., Jones, Hanton, \& Connaughton, 2002). Member checking was used to ensure robustness of the data, whereby each participant was given a copy of their transcribed interview and asked to confirm its accuracy (Jones et al., 2002).

\section{Instrumentation: Psychological momentum measure}

Due to the timing of the access granted, the new PM measure was developed using the "raw themes" $(N=61)$ generated from previous interviews conducted regarding players' perceptions of PM in soccer (Jones \& Harwood, 2008). Minor modifications were guided by discussion and feedback from coaches, athletes, and experts (e.g., football managers and academics with more than 15 years of experience). The resulting 49 items comprised of four sub-scales including; triggers of positive PM $(n=14)$; triggers of negative $\mathrm{PM}(n=14)$; strategies to maintain positive PM $(n=15)$ and strategies to overcome negative $\mathrm{PM}(n=6)$. As with previous measures used to assess athletes' experiences and behaviours (Lonsdale, Hodge, \& Rose, 2008), players used a 7-point scale $(1=$ not at all and $7=$ very much so $)$ to rate the extent that the item applied to their own experiences. Players were encouraged to be open and honest in their responses, and that there were no right or wrong answers.

\section{Data analysis: Qualitative}

Interview and focus group data were manually analysed using a combination of inductive and deductive approaches (see Patton, 2002). The previously identified four dimensions; experiences of positive PM, which included both triggers and characteristics, experiences of negative PM, which included both triggers and characteristics, strategies to 
gain or maintain positive momentum, and strategies to overcome negative momentum were used to guide the deductive analysis process, but there was flexibility for discovering new factors, themes, and/or dimensions from the current investigation. For inductive analysis, the primary investigator read and re-read 40 pages of transcribed verbatim individual interviews and four pages of focus group interview data several times to ensure familiarity and promote accuracy of data interpretation. Given that focus groups did not yield additional themes and, instead, provided clarification and useful insight to previous discussions with interviewees, qualitative data analysis was derived from the content of individual interviews $(N=10)$ only. Based on principles of inductive qualitative analysis as identified by Patton (2002), themes were categorised using a four-stage process: (a) Raw data were systematically extracted from transcriptions. (b) Raw data were then clustered into units according to similarity of meaning. (c) First order themes were then established through further systematic organisation of broader, overarching concepts. (d) Finally, higher order themes were ultimately positioned by common factors according to the four previously-specified dimensions. Although the current study yielded more first order and higher order themes compared to findings of Jones and Harwood (2008), no new dimensions were identified.

\section{Trustworthiness of data analysis}

One of the co-authors, who was both familiar with PM research and experienced in qualitative data analysis, was presented with $25 \%$ of the transcribed data and the entirety of the extracted raw data, first order themes, and higher order themes for the purpose of verification. Similar to the process used by Mellalieu, Neil, Hanton, and Fletcher (2009), the second researcher acted as "devil's advocate" to read and re-read the findings and to discuss the initial analysis conducted by the principal researcher. For each stage of analysis, feedback from the second researcher informed discussions with the primary researcher, and revisions 
were made accordingly. The two authors met regularly during the analysis to check coding decisions and to check the emerging higher order themes.

The reliability of the coding process was assessed in line with the methods used by Briki, Den Hartigh, Hauw, and Gernigon (2012). Agreement rating [(number of same findings)/(number of same findings + number of divergent findings)] was calculated resulting in agreement ratings of $86.9 \%, 85 \%$ and $87.5 \%$ for raw themes, $1^{\text {st }}$ order themes and higher order themes, respectively. Reliability for all three inter-coder agreements were above the minimal percentage of $70 \%$ as recommended by Van Someren, Barnard, and Sandleberg (1994).

\section{Data analysis: Quantitative}

A series of five Chi-square tests of independence was performed to assess differences between responses to the 49 questions both individually and for the set of questions as a whole. Alpha was set at $p<.05$ for all statistical tests. In line with recommendations of Fallowfield, Hale, and Wilkinson (2005) for two of the four dimensions (triggers of positive PM and strategies to overcome negative momentum), frequency data for the first two points (1 and 2) of the 7-point Likert scale were combined when performing Chi-square tests to ensure that the data satisfied the assumption of an expected frequency of at least 5.0 in at least $80 \%$ of the cells.

\section{Results: Qualitative Analysis}

Inductive content analysis yielded a total of 848 raw data extracts (see supplementary Tables A-D for raw data extracts) from the verbatim transcriptions across the four dimensions of PM (players' experiences of positive PM $(n=300)$; experiences of negative momentum ( $n$ $=193)$; players' strategies for maintaining positive momentum $(n=108)$; strategies to overcome negative momentum $(n=247))$. These were then categorised into first order $(n=$ 58) and, in turn, higher order $(n=24)$ themes according to the four above specified 
dimensions. When reporting the interview data, higher order themes are listed at the beginning of each section in descending order (based on frequency totals in parentheses; see Figures $1 \& 2$ ), followed by key first order themes, and finally their respective supporting quotes (coded as P1, P2...P10 throughout) in order to contextualise the depth and finer detail of this research.

\section{Experiences of Positive Psychological Momentum}

Players revealed several factors associated with experiences of positive PM, including performance factors, external influences, confidence, general feelings and emotions, group factors, and recognise the need for PM (Figure 1). When considering performance factors, all players interviewed associated experiences of positive momentum with scoring a goal; for example, a player explained that, "If the team has just scored, they feel a lot more confident and positive, especially when they see their opponents' heads go down" (P10).

Within the higher order themes of general feelings and emotions and confidence, the first order themes of positive feelings and confidence were highly salient features for the players, as evidenced by their prevalence and the depth of discussion by players. For example, players noted, "When you have momentum you feel confident, you are positive and your whole attitude changes. You want to score and it's likely" (P8), and, "when it's going our way and we are on top, we feel good, we feel more confident and composed on the ball lots of positive thoughts" (P4).

\section{Experiences of Negative Psychological Momentum}

The perception of negative PM was associated with a number of interesting factors, categorised under the following higher order themes; psychological aspects of negative PM, group experiences of negative PM, confidence issues, negative effects on performance, external influences, and positive effects on performance (Figure 1). Similar to positive experiences of PM, within the psychological aspects higher order theme, feelings and 
External Influence Coach/

Manager/Captain (19)

Crowd (12)

Opponents (12)

Referee (7)

Encouragement (25)

Recognize the need for PM (2)

Effort (10)

Starting the game (10)

Scoring (42)

Individual performance (20)

Positive feelings (51)

No pressure calm (3)
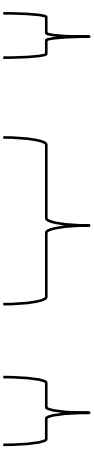

Recognize the need for PM (2)

Performance Factors (82)

General Feelings \& Emotions (54)

Influences (75)

Confidence (64)

pper hand (10)

Confidence (35)

Positive body language/attitude (19)

Group efficacy (6)

Teamwork (11)

Group performance (6)

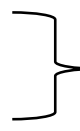

Group Factors (23)

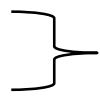

Psychological

Aspects of PM (85)

Feelings and emotions (61)

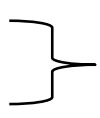

Group Experiences of Negative PM (31)

Negativity within team (23)

$$
\text { of Negative PM (31) }
$$

Facilitates performance (20)

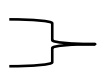

Crowd Effects (3)

Opponent Effects (5)

Referee (3)

Conceding (2)

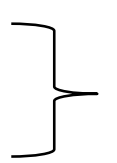

Positive Effects on

Performance (20)

External

Influences (13)

Players

Experiences

of Positive

PM (300)
Players

Experiences

of Negative

PM (193)

Loss of Confidence (18)

Ability (7)

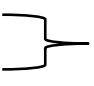

Confidence

Issues (25)

Debilitates Performance (12)

Effort (7)

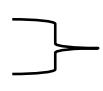

Negative Effects on -

Performance (19)

Figure 1: Inductive Analysis: Players Experiences of PM (N=10) 
emotions and body language were both first order themes that were highly salient features for the interviewed players. Specifically, players frequently reported visible changes in their body language (and confidence) when they conceded a goal with 'heads going down (when you concede)' the most frequently reported raw data extract. In addition, there was a wide range of feelings and emotions that players associated with experiences of negative momentum such as feeling under pressure, and being worried, nervous, and angry. For example, one player recalled, "[When you perceive momentum is against you,] head goes down, 50/50, [you feel] frustrated, angry, feel fatigued and under pressure” (P9).

Within group experiences of negative PM, players commonly referred to their negative interactions with other teammates when they perceived that momentum was not on their side; for example; "[When momentum is against us, the] communication goes out the window, negative comments like shouting at each other arguing, like everyone is against you blaming you" (P4).

\section{Strategies for Building and Maintaining Psychological Momentum}

Strategies used to maintain and develop positive PM included tactical, technical, and individual strategies, being better prepared, and teamwork (Figure 2). More so than the other three dimensions, this area provided a wide range of raw data relative to its first order themes, suggesting that players use a variety of strategies to maintain and develop positive PM. Various technical and tactical related factors (e.g., keeping possession of the ball and making more passes) and use of psychological skills (e.g., maintaining concentration and focus) were highly prevalent in comparison to other factors or strategies, and overall elicited a far greater depth of discussion in the individual interviews. 
Tactical Objective (14)

Tactical Strategy (General) (5)

Tactical Strategy (Individual) (13)

Tactical Choice (15)

Technical Choice (22)

Technical Objective (7)

Psychological Skills

Mind-Set (14)

Increasing Effort

(Individually) (6)

Teamwork (3)

Fitness (5)

Preparation (4)

Staying Positive (39)

Don't let it get to you (9)

Just keep going (26)

Opponents (11)

Crowd (7)

Encouragement (33)

Teamwork (22)

Effort (26)

Tactical Objective (6)

Opponents (tactical) (6)

Controlling Pace (13)

Technical Choice (13)

Technical Objective (14)

Psychological Skills (15)

First Chances (2)

Performance (positive) (5)
Tactical

Strategies (47)

Technical

Strategies (29)

Individual

Strategies (20)

Teamwork (3)

Being Better

Prepared (9)
Strategies Used

to Maintain/Gain

Positive PM (108)
Strategies Used

To Overcome

Negative

PM (247)

Figure 2: Inductive Analysis: Strategies Used to Maintain/Gain/Overcome PM ( $N=10)$ 
With regard to psychological skills, keeping focused and maintaining concentration were reported as strategies used frequently by players especially after scoring; for example, a player noted:

I feel [that] especially after we score, we need to be focused, because as everyone knows this is the time when you are most vulnerable as people tend to switch off (P1).

\section{Strategies for Overcoming Negative Psychological Momentum}

Players revealed several strategies used to overcome negative momentum, including keeping a positive attitude, external influences, technical strategies, increasing effort, tactical strategies, performance strategies, and working as a team (Figure 2). Within the higher order themes of keeping a positive attitude and external influences, the first order themes of staying positive and keeping going, and encouragement from teammates and coaches were highly salient features for the players. For example, players frequently noted, the importance of looking and feeling optimistic, "[When you start to lose momentum] keep your head up, keep tracking back, be more positive" (P9).

With regard to encouragement, players noted, “[You're always] telling them 'keep head up', keep on going, don't give up, you got him, and keep putting 100\% in" (P6). In terms of keeping going, a number of players reported it was important to "Keep doing what you can" (P7) in order to overcome negative momentum.

\section{Results: Quantitative Analysis}

The PM measure was completed by 75 participants. Descriptive statistics from their responses are presented in Tables 1 and 2.Chi-square results highlight differences within each of the four dimensions when analysed individually (triggers of positive momentum $\chi^{2}(65, N$ $=75)=449.0, \mathrm{p}<.001)$, triggers of negative momentum $\chi^{2}(78, N=75)=376.5, \mathrm{p}<.001$, strategies used to maintain positive momentum $\chi^{2}(78, N=75)=445.7, \mathrm{p}<.001$, and 
Table 1:

Questionnaire Responses for Players' Experiences of PM (N=75)

\begin{tabular}{|c|c|c|c|c|c|c|c|c|c|c|c|c|c|}
\hline Experience of positive PM & Question & 1 & 2 & 3 & 4 & 5 & 6 & 7 & Mean & SD & Median & Mode & Rank \\
\hline You or your team score a goal & 6 & 0 & 0 & 0 & 3 & 5 & 18 & 49 & 6.51 & 0.79 & 7 & 7 & 1 \\
\hline You feel confidence & 4 & 0 & 0 & 0 & 1 & 11 & 26 & 37 & 6.32 & 0.77 & 6 & 7 & 2 \\
\hline The team feels cohesion/united & 13 & 1 & 0 & 3 & 10 & 18 & 21 & 22 & 5.60 & 1.27 & 6 & 7 & 3 \\
\hline You have a positive attitude & 14 & 0 & 1 & 3 & 10 & 17 & 24 & 20 & 5.60 & 1.21 & 6 & 6 & 3 \\
\hline Opponents make mistakes & 3 & 3 & 4 & 1 & 5 & 18 & 25 & 18 & 5.41 & 1.56 & 6 & 6 & 5 \\
\hline Opponents weaknesses are highlighted & 2 & 0 & 3 & 5 & 10 & 20 & 16 & 21 & 5.39 & 1.40 & 5 & 7 & 6 \\
\hline You see negative body language from opponents & 1 & 0 & 2 & 6 & 12 & 20 & 18 & 17 & 5.29 & 1.34 & 5 & 5 & 7 \\
\hline You receive encouragement from coach & 10 & 0 & 2 & 4 & 16 & 24 & 23 & 6 & 5.07 & 1.14 & 5 & 5 & 8 \\
\hline You receive encouragement from teammates & 9 & 0 & 1 & 10 & 17 & 27 & 17 & 3 & 4.77 & 1.11 & 5 & 5 & 9 \\
\hline Good luck/fortune & 5 & 5 & 8 & 7 & 19 & 15 & 14 & 7 & 4.35 & 1.68 & 4 & 4 & 10 \\
\hline Referees decisions going your way & 7 & 3 & 5 & 11 & 24 & 19 & 11 & 2 & 4.23 & 1.35 & 4 & 4 & 11 \\
\hline Previous experiences/achievements & 8 & 8 & 11 & 6 & 12 & 17 & 16 & 5 & 4.16 & 1.82 & 5 & 5 & 12 \\
\hline You receive encouragement from crowd & 11 & 8 & 14 & 14 & 14 & 14 & 9 & 2 & 3.63 & 1.64 & 4 & $2,3,4,5$ & 13 \\
\hline You receive encouragement from captain & 12 & 8 & 16 & 13 & 17 & 14 & 6 & 1 & 3.47 & 1.54 & 4 & 4 & 14 \\
\hline Experience of negative PM & Question & 1 & 2 & 3 & 4 & 5 & 6 & 7 & Mean & SD & Median & Mode & Rank \\
\hline You or your team concede a goal & 20 & 0 & 0 & 0 & 1 & 18 & 24 & 32 & 6.16 & 0.84 & 6 & 7 & 1 \\
\hline Lack of perceived ability & 25 & 1 & 2 & 4 & 5 & 19 & 27 & 17 & 5.51 & 1.33 & 6 & 6 & 2 \\
\hline Feeling nervous and/or anxious & 24 & 2 & 2 & 4 & 13 & 15 & 24 & 15 & 5.25 & 1.46 & 6 & 6 & 3 \\
\hline When you feel fatigue & 26 & 1 & 3 & 5 & 12 & 21 & 18 & 15 & 5.17 & 1.43 & 5 & 5 & 4 \\
\hline Loss of concentration & 23 & 2 & 1 & 6 & 16 & 17 & 21 & 12 & 5.08 & 1.42 & 5 & 6 & 5 \\
\hline Bad luck/fortune & 19 & 1 & 8 & 5 & 13 & 21 & 17 & 10 & 4.81 & 1.54 & 5 & 5 & 6 \\
\hline Uncontrollable response of opponent & 18 & 1 & 3 & 11 & 19 & 18 & 11 & 12 & 4.75 & 1.47 & 5 & 4 & 7 \\
\hline Bad Refereeing decisions & 21 & 1 & 6 & 7 & 19 & 16 & 20 & 6 & 4.69 & 1.44 & 5 & 6 & 8 \\
\hline Feelings that members of team are being complacent & 22 & 1 & 3 & 12 & 18 & 21 & 15 & 5 & 4.60 & 1.34 & 5 & 5 & 9 \\
\hline When you feel under pressure & 28 & 4 & 7 & 10 & 16 & 18 & 10 & 10 & 4.43 & 1.68 & 5 & 5 & 10 \\
\hline Negative criticism & 27 & 4 & 8 & 9 & 22 & 13 & 17 & 2 & 4.21 & 1.53 & 4 & 4 & 11 \\
\hline Playing opponents of a higher ability & 15 & 8 & 8 & 11 & 17 & 10 & 13 & 8 & 4.12 & 1.83 & 4 & 4 & 12 \\
\hline Opponents strengths are highlighted & 16 & 18 & 14 & 14 & 16 & 9 & 4 & 0 & 2.95 & 1.53 & 3 & 1 & 13 \\
\hline Opponents reputation & 17 & 17 & 15 & 22 & 13 & 5 & 3 & 0 & 2.77 & 1.37 & 3 & 3 & 14 \\
\hline
\end{tabular}

Note. Table is arranged by descending mean scores according to each dimension. 
Table 2:

Questionnaire Reponses for Players' Experiences of PM - Strategies $(N=75)$

\begin{tabular}{|c|c|c|c|c|c|c|c|c|c|c|c|c|c|}
\hline Strategies to maintain/create positive PM & Questio & 1 & 2 & 3 & 4 & 5 & 6 & 7 & Mean & SD & Median & Mod & Rank \\
\hline Preparation & 30 & 0 & 3 & 1 & 5 & 19 & 23 & 24 & 5.73 & 1.24 & 6 & 7 & 3 \\
\hline Retaining possession & 41 & 0 & 2 & 4 & 12 & 20 & 22 & 15 & 5.35 & 1.27 & 5 & 6 & 5 \\
\hline Targeting opponent's weaknesses & 43 & 0 & 3 & 4 & 15 & 13 & 22 & 18 & 5.35 & 1.39 & 6 & 6 & 5 \\
\hline Giving encouragement to team mates & 42 & 3 & 4 & 4 & 7 & 18 & 18 & 21 & 5.28 & 1.66 & 6 & 7 & 7 \\
\hline Use of time (slowing down/speeding up the game) & 35 & 3 & 5 & 8 & 15 & 16 & 17 & 11 & 4.75 & 1.63 & 5 & 6 & 10 \\
\hline Goal setting & 29 & 7 & 8 & 6 & 19 & 16 & 12 & 7 & 4.24 & 1.74 & 4 & 4 & 11 \\
\hline Changing tactics & 34 & 1 & 7 & 8 & 15 & 17 & 10 & 7 & 4.04 & 1.86 & 4 & 5 & 12 \\
\hline Going back to basics & 36 & 1 & 11 & 1 & 12 & 10 & 6 & 5 & 3.31 & 1.89 & 3 & 1 & 13 \\
\hline Using confidence building strategies & 37 & 1 & 17 & 7 & 15 & 6 & 8 & 5 & 3.27 & 1.91 & 3 & 1,2 & 14 \\
\hline Trigger words (which help to stay focused, & 32 & 2 & 20 & 9 & 11 & 4 & 5 & 3 & 2.73 & 1.75 & 2 & 1 & 15 \\
\hline Frustrating opponents & 46 & 2 & 5 & 7 & 14 & 15 & 19 & 13 & 4.92 & 1.60 & 5 & 6 & 3 \\
\hline Managing pressure & 47 & 5 & 3 & 8 & 10 & 15 & 23 & 11 & 4.87 & 1.70 & 5 & 6 & 4 \\
\hline Controlling pace & 45 & 0 & 5 & 7 & 20 & 17 & 20 & 6 & 4.77 & 1.34 & 5 & 4,5 & 5 \\
\hline Managing anxiety & 48 & 8 & 8 & 1 & 12 & 9 & 16 & 10 & 4.25 & 1.92 & 4 & 6 & 6 \\
\hline
\end{tabular}

Note. Table is arranged by descending mean scores according to each dimension. 
strategies used to overcome negative momentum $\left.\chi^{2}(25, N=75)=44.72, \mathrm{p}<.001\right)$, and also for the set of 49 questions as a whole $\left.\chi^{2}(228, N=75)=1440.64, \mathrm{p}<.001\right)$. Thus, differences were found between the distribution of responses across both individual questions and the four specified dimensions (e.g., some statements/dimensions apply to the 75 players more than others).

\section{Discussion}

The purpose of the current study was to investigate elite soccer players' experiences (triggers, characteristics and strategies) of psychological momentum (PM) using a mixed methodological approach. As the majority of experimental studies have not focused on the different kinds of triggers and strategies of PM, the quantitative methods aimed to provide further insight into this aspect of PM. A number of the experiences reported were highlighted by players in their interviews as both triggers and effects of PM. For this reason, unlike previous models (Taylor \& Demick, 1994; Vallerand et al., 1988) which have separated these experiences, the current study reports on players' experiences combined with a focus on triggers of PM, specifically those that were consistent across both methods. Strategies highlighted as important to either maintaining/creating positive PM or overcoming negative PM are discussed separately due to variation across players.

The act of scoring or conceding a goal yielded the highest average scores from players' questionnaire responses for positive and negative PM. This is in agreement with previous research that has highlighted the importance of goal scoring (Gernigon et al., 2010; Higham et al., 2005; Jones \& Harwood, 2008) and positive score configuration (Briki et al., 2013; Stanimirovic \& Hanrahan, 2004; Vallerand et al., 1988) in relation to PM experiences. Scoring a goal was also reported most frequently within the performance factors higher order theme by players interviewed. Indeed, all players interviewed referred to goal scoring as a 
factor related to positive PM, reporting that a positive PM state would often result in progression toward a goal/scoring and that progressing toward a goal/scoring also triggered positive PM. With regards to negative PM, body language - specifically when athletes described their heads going down after conceding a goal - accounted for $12.4 \%$ of all raw data extracts in player interviews. Although goal scoring was highlighted by Jones and Harwood (2008) as a trigger of positive PM, it is difficult to establish the strength of its importance as the authors, unlike the current study, provided no details with regard to the number of respondents who reported each theme.

Feeling confident was also an important factor related to positive PM and was reported second-most frequently across player interviews as both a trigger and characteristic of positive PM, and this also yielded the second highest questionnaire average score. Jones and Harwood (2008) found confidence to be the most frequently cited trigger of PM in their interviews with soccer players. A number of other studies have also found self-confidence to increase in positive PM scenerios and decrease when participants experience negative PM (Briki, Den Hartigh, Bakker, \& Gernigon, 2012; Den Hartigh et al., 2014). Both Briki, Den Hartigh, Hauw, and Gernigon (2012) and Gernigon et al. (2010) found that progressing toward (or away from) a desired outcome (e.g., victory or defeat) increased (decreased) selfconfidence. This further supports the prominent findings regarding scoring in the current study, especially as having a lack of ability was reported as the second highest average score related to negative PM on the PM measure.

Although confidence was an important aspect of positive PM in the current study, its importance was proceeded by goal scoring, unlike previous studies which have found selfconfidence (Jones \& Harwood, 2008) and opponent ability (Moesch \& Apitzsch, 2012) to be the most salient factor relating to PM experiences. It is plausible to suggest that the prescence of self-confidence in the current study may facilitate the development of PM but also that a 
positive PM scenerio may enhance self-efficacy at the same time - especially as teams progress toward their goal. Recently Den Hartigh, Van Geert, Van Yperen, Cox, and Gernigon (2016) demonstrated that athletes who enter a match with relatively high levels of self-efficacy were better able to maintain their levels of self-efficacy, PM perception, and efforts whilst in a negative PM scenerio. It is possible that the experienced players recruited in the current study may be better able to moderate PM perceptions compared to the less experienced athletes interviewed by Jones and Harwood (2008).

It would seem that confidence, experience, and belief in one's ability are key factors affecting how PM is perceived, and from a practical perspective, should be developed through positive reinforcement and mastery during training sessions (Vallerand \& Reid, 1984). The use of confidence-building strategies, however, was reported as one of the least popular strategies for maintaining positive PM in the player measure, giving further support for the notion that players at this level may have a lower need to build confidence as they already have a high level of perceived ability. Maintaining concentration was the highest reported strategy for maintaining PM; therefore, it may be more advisable for coaches to channel energy into strategies that focus attention on creating positive PM.

Supporting previous studies (Jones \& Harwood, 2008; Moesch \& Apitzsch, 2012), results from the current study indicate that having a positive attitude was one of the highest reported experiences of positive PM in the player questionnaires, and it was frequently cited in player interviews. Interestingly, although feeling anxious/nervous was reported as one of the highest experiences of negative PM, managing anxiety as a strategy was the lowest reported item on the measure with regard to strategies to overcome negative PM. It would appear that although increases in competitive anxiety are related to negative PM and decreases to positive PM (Briki, Den Hartigh, Bakker, \& Gernigon, 2012; Gernigon et al., 2010), there may be a number of factors that interact in order for either positive or negative PM to evolve. This 
provides further support for Gernigon et al.'s (2010) suggestion that PM manifests itself as a series of complex interactions that create changes in behaviour. It may therefore be more appropriate for coaches to focus on strategies that maximise the chances of success (e.g., adequate preparation, which was reported one of the highest strategies by the PM measure) as a way to perhaps mediate unfavourable scenarios.

Although research often indicates the detrimental effects of negative PM on performance (Stanimirovic \& Hanranhan, 2004; Taylor \& Demick, 1994), the findings from this study show that experiencing negative PM actually aided performance. Notably, this was also found by Perreault et al. (1998) who concluded that losing and experiencing negative momentum did not necessarily result in a decrease in performance. The latter findings, in particular, support the concept of negative facilitation (Cornelius et al., 1997; Stanimirovic \& Hanrahan, 2004), whereby performers work harder when they perceive that they are behind. More recently however, studies have found that although athletes may increase their efforts when experiencing negative momentum, this gradually decreases once they are convinced they will lose (e.g., after a period of resisting negative PM) (Briki, Den Hartigh, Hauw, \& Gernigon, 2012; Briki et al., 2013). This could explain why some studies have found that performers increase their efforts immediately after conceding in the hope of scoring on the counterattack (Redwood-Brown, 2008; Ridgewell, 2011).

For maintaining positive PM, athletes' questionnaire responses indicated the most frequently reported strategy was maintaining concentration which was also reported by players interviewed. Being prepared was also reported as an important strategy for creating positive momentum, with physical preparation (training preparation) and preparation representing the second and third highest mean scores from the questionnaire, respectively. Similarly, Jones and Harwood (2008) reported that feeling prepared put players in a more positive frame of mind and helped them to gain momentum and develop a positive attitude, 
which has already been established as key to positive PM experiences. Coaches interviewed by Moesch and Apitzsch (2012) considered being well-prepared to be a concentration enhancing strategy that could also be used during matches to build PM or refocus. It is important, therefore, that coaches understand the importance of perceived preparation especially as these two factors were also the most frequently reported strategies in the current study to maintain positive PM.

Changing tactics was the highest reported strategy from the questionnaires for overcoming negative PM with tactical and technical strategies featuring frequently in player interviews. Interestingly, controlling the game by keeping possession has been shown as a key attribute of successful soccer teams (Jones, James, \& Mellalieu, 2004) and was a key strategy reported by players interviewed by Jones and Harwood (2008) for overcoming negative PM. Players and coaches should also practice changing tactics to overturn the balance of momentum (if in a negative PM state) in training matches, as the change itself maybe more important than any particular tactic enforced (Higham et al., 2005).

Coaches and managers need to be aware of not only what triggers the momentum changes, but also when momentum may be changing. They need to recognise when the flow may be starting to go against them and make the necessary changes to avoid this happening (e.g., change tactics or make a change to the line-up through a substitution or play). For example, in volleyball, Salitsky (1995) observed that following a time-out, the opposition with positive psychological momentum reduced the number of points scored. Having possession (e.g., feeling superior/in control) may act as a similar strategy and allow a team to re-configure after a period of negative momentum or a drop in performance (Higham et al., 2005). Knowing when momentum is most likely to change (and what causes it to change) could have implications on how coaches and players employ strategies to create positive and overcome negative PM. 
In terms of external strategies, athletes frequently reported encouragement, specifically from teammates, as a valuable strategy used to overcome negative PM. Similarly, on the questionnaire, encouragement from teammates yielded the second highest average score within this dimension. Den Hartigh et al. (2014) found team cohesion increased alongside collectiveefficacy when teams experienced positive PM, but decreased with negative PM. It is possible that teams who have a strong sense of unity and support are able to use such encouragement to foster a positive outlook to overcome negative PM or produce positive momentum (Vallerand et al., 1988). Facilitating positive group dynamics within team training sessions where negative momentum is experienced may also be a useful way to help players create team strategies to cope with such situations, especially as teams who are cohesive and confident are more likely to remain united in the face of failure and vice versa (Stanimirovic \& Hanrahan, 2004). Coaches have also reported not sticking to one's role and not taking responsibility as key actions for the onset of negative PM (Moesch \& Apitzsch, 2012). Ensuring team goals and roles are set out clearly and with measurable targets may be one strategy for coaches to adopt to ensure teams remain on target and focused on the desired outcome.

\section{Limitations}

The current study extends the qualitative work of researchers who have attempted to gain an understanding of the perceptions of PM in sport. Although only one team was used in the qualitative part of the study, the use of quantitative methods greatly increased the number of players who reported their experiences and perceptions of PM. However, using a case study approach with only one team may have influenced players' responses as all players were coached by the same coaching team and were drilled in the same manner, and therefore may share similar perceptions with regard to success and failure. Although individual perceptions would still occur, this does not allow for the data to be generalised across teams. Individual player data analysis was also limited as only the accumulated player responses 
were recorded within the PM measure. Future research should therefore consider using more than one team for individual player interviews, and also compare responses across a number of positions and levels to provide a clearer picture of the entire team, each player's PM experiences, and subsequent strategies that can be used to control PM.

The current study built upon previous research by including athletes' experiences of PM across multiple teams, however there was no account for match outcome (e.g., after a win or after a loss), which may have positively or adversely influenced athletes' responses, especially as this has been a key feature of a number of studies. Conducting both interviews and questionnaires with players following different match outcomes may help to eradicate this problem as well as to establish how momentum manifests itself in different score line conditions.

\section{Conclusion}

In conclusion, this study provides a valuable insight into the key experiences of PM and the strategies associated with positive and negative PM. The majority of responses from the PM measure were supported by the responses from the player interviews. Although previous experimental studies have shown progression toward or away from a desired goal to affect perceptions of PM, the findings in the current study were based on individual experiences rather than manipulated performance scenarios where all participants were subject to the same kind of manipulation (progression or regression). Importantly, the individualised nature of PM perceptions (Markman \& Guenther, 2007; Vallerand et al., 1988) was supported throughout, with players reporting a wide range of strategies to either maintain positive PM or overcome negative PM.

It was also proposed that PM experiences may be dependent on not only the ability of individuals but also the sport in question. Such findings highlight the need for further investigation across multiple age groups, abilities, and sexes using larger sample sizes, and 
this could be facilitated by quantitative methods such as those used in the current study. Whilst further investigation is needed to establish the questionnaire as a valuable tool to assess players' PM perceptions, the findings from the sample of soccer players' questionnaires were largely supported by player interviews (e.g., the most salient qualitative themes were also the most frequently reported questionnaire responses). This therefore highlights the questionnaire as a useful tool in gathering data on players' experiences of PM within soccer in closer proximity to players actually experiencing PM, to enable both coaches and players to maximise positive PM, reduce negative PM, and develop strategies to optimise PM.

\section{References}

Adler, P. (1981). Momentum: A theory of social action. Beverly Hills, CA: Sage.

Bradley, J., \& O’Donoghue, P. G. (2011). Counterattacks in elite Gaelic football competition. International Journal of Performance Analysis in Sport, 11,159-170.

Briki, W., Den Hartigh, R. J., Hauw, D., \& Gernigon, C. (2012). A qualitative exploration of the psychological contents and dynamics of momentum in sport. International Journal of Sport Psychology, 43(5), 365-384.

Briki, W., Den Hartigh, R. J., Bakker, F.C., \& Gernigon, C. (2012). The dynamics of psychological momentum: A quantitative study in natural sport situations. International Journal of Performance Analysis in Sport, 12, 573-592.

Briki, W., Den Hartigh, R. J., Markman, K. D., Micallef, J. P., \& Gernigon, C. (2013). How psychological momentum changes in athletes during a sport competition. Psychology of Sport and Exercise, 14(3), 389-396. doi: 10.1016/j.psychsport.2012.11.009.

Burke, K. L., Edwards, T. C., Weigand, D. A., \& Weinberg, R. S. (1997). Momentum in sport: A real or illusionary phenomenon for spectators. International Journal of Sport Psychology, 28, 79-96. 
Crust, L., \& Nesti, M. (2006). A review of psychological momentum in sports: Why qualitative research is needed. Athletic Insight, 8, 1-15.

Cornelius, A. E., Silva, J. M., Conroy, D. E., \& Petersen, G. (1997). The projected performance model: Relating cognitive and performance antecedents of psychological momentum. Perceptual and Motor Skills, 84, 475-485. doi: 10.2466/pms.1997.84.2.475.

Den Hartigh, R. J., Gernigon, C., Van Yperen, N. W., Marin, L., \& Van Geert, P. L. (2014). How psychological and behavioral team states change during positive and negative momentum. PloS one, 9(5), e97887. doi: 10.1371/journal.pone.0097887.

Den Hartigh, R. J., Van Geert, P. L., Van Yperen, N. W., Cox, R. F., \& Gernigon, C. (2016). Psychological Momentum During and Across Sports Matches: Evidence for Interconnected Time Scales. Journal of Sport \& Exercise Psychology, 38(1), 82-92. doi: 10.1123/jsep.2015-0162.

Eisler, L., \& Spink, K. S. (1998). Effects of scoring configuration and task cohesion on the perception of psychological momentum. Journal of Sport \& Exercise Psychology, 20, 311-320.

Fallowfield, J. L., Hale, B. J., \& Wilkinson, D. M. (2005). Using Statistics in Sport and Exercise Research, Chichester, UK: Lotus Publishing.

Ford, P. R., \& Williams, M. A. (2011). No relative age effect in the birth dates of awardwinning athletes in male professional team sports. Research Quarterly for Exercise and Sport, 82(3), 570-573.

Gernigon, C., Briki, W., \& Eykens, K. (2010). The dynamics of psychological momentum in sport: The role of ongoing history of performance patterns. Journal of Sport \& Exercise Psychology, 32(3), 377. doi: 10.1123/jsep.32.3.377 
Hanton, S., Fletcher, D., \& Coughlan, G. (2005). Stress in elite sports performers: A qualitative investigation of competition and organizational stressors. Journal of Sports Sciences, 10, 1129-1141. doi: 10.1080/02640410500131480.

Higham, A., Harwood, C., \& Cale, A. (2005). Momentum in Soccer: Controlling the Game. Leeds: Coachwise/1st for Sport Publications.

Hoffmann, R., Ging, L. C., \& Ramasamy, B. (2002). The socio-economic determinants of international soccer performance. Journal of Applied Economics, 5(2), 253-257.

Iso-Ahola, S. E., \& Dotson, C. O. (2014). Psychological momentum: Why success breeds success. Review of General Psychology, 18(1), 19. doi: 10.1037/a0036406.

Jones, G., Hanton, S., \& Connaughton, D. (2002). What is this thing called mental toughness? An investigation of elite sport performers. Journal of Applied Sport Psychology, 14, $205-$ 218. doi: 10.1080/10413200290103509.

Jones, M. I., \& Harwood, C. (2008). Psychological momentum within competitive soccer: Players' perspectives. Journal of Applied Sport Psychology, 20, 57-72. doi:10.1080/10413200701784841.

Jones, P. D., James, N., \& Mellalieu, S. D. (2004). Possession as a performance indicator in soccer. International Journal of Performance Analysis of Sport, 4, 98-102.

Koehler, J. J., \& Conley, C. A. (2003). The "hot hand" myth in professional basketball. Journal of Sport and Exercise Psychology, 25, 253-259. doi: 10.1123/jsep.25.2.253.

Lonsdale, C., Hodge, K., \& Rose, E. A. (2008). The behavioural regulation in sport questionnaire (BRSQ): Instrument development and initial validity evidence. Journal of Sport and Exercise Psychology, 30, 323-335. doi: 10.1123/jsep.30.3.323.

Markman, K. D., \& Guenther, C. L. (2007). Psychological momentum: Intuitive physics and naïve beliefs. Personality and Social Psychology Bulletin. doi: 10.1177/0146167207301026 
Mellalieu, S. D., Neil, R., Hanton, S., \& Fletcher, D. (2009). Competition stress in sport performers: Stressors experienced in the competition environment. Journal of Sports Sciences, 27, 729-744. doi: 10.1080/02640410902889834.

Moesch, K., \& Apitzsch, E. (2012). How do coaches experience psychological momentum? A qualitative study of female elite handball teams. Sport Psychologist, 26(3), 435. doi: 10.1123/tsp.26.3.435.

Patton, M. Q. (2002). Qualitative Research and Education Methods. Thousand Oaks, CA:Sage. Perreault, S., Vallerand, R. J., Montgomery, D., \& Provencher, P. (1998). Coming from behind: On the effect of psychological momentum on sport performance. Journal of Sport and Exercise Psychology, 20,421-436. doi: 10.1123/jsep.20.4.421.

Redwood-Brown, A. (2008). Passing patterns before and after goal scoring in FA Premier League Soccer. International Journal of Performance Analysis in Sport, 8(3), 172-182.

Ridgewell, A. (2011). Passing patterns before and after scoring in the 2010 FIFA World Cup. International Journal of Performance Analysis in Sport, 11(3), 562-574.

Salitsky, P. B. (1995). Effects of time-outs on psychological momentum in intercollegiate women's volleyball. Microform Publications, University of Oregon.

Stanimirovic, R., \& Hanrahan, S. J. (2004). Efficacy, affect, and teams: Is momentum a misnomer? International Journal of Sport and Exercise Psychology, 2(1), 43-62. doi: 10.1080/1612197x.2004.9671732.

Taylor, J., \& Demick, A. (1994). A multi-dimensional model of momentum in sports. Journal of Applied Sport Psychology, 6, 51-70. doi: 10.1080/10413209408406465.

Vallerand, R. J., \& Reid, G. (1984). On the causal effect of perceived competence on intrinsic motivation: A test of cognitive evaluation theory. Journal of Sport Psychology, 6,94-102. doi: org/10.1123/jsp.6.1.94. 
Vallerand, R. J., Colavecchio, P. G., \& Pelletier, L. G. (1988). Psychological momentum and performance influences: a preliminary test of the antecedents' consequences psychological momentum model. Journal of Sport and Exercise Psychology, 10, 92-108. doi :10.1123/jsep.10.1.92.

Someren, M. V., Barnard, Y. F., \& Sandberg, J. A. (1994). The Think Aloud Method: A Practical Approach to Modelling Cognitive Processes. Academic Press. 